\section{Le paradis des listes de vérification : le réseau EQUATOR}

Notre Unité de recherche en pratique pharmaceutique célèbre son $10^{\mathrm{e}}$ anniversaire cette année'. Après une décennie de recul, force est de constater les difficultés inhérentes à la recherche portant sur les modèles et les pratiques pharmaceutiques : formation de $1^{\text {er }}$ cycle insuffisante pour la réalisation d'un projet de recherche, formation de $2^{e}$ cycle (maitrise en pharmacothérapie avancée) limitée à une initiation à la recherche, nombre restreint de professeurs et de chercheurs intéressés à cette thématique, sources de financement limitées, etc. Charrois et coll. ont aussi mis en évidence ces difficultés dans le cadre d'une revue systématique concernant la recherche en pratique pharmaceutique ${ }^{2}$.

Dans la foulée de la réflexion menant à la rédaction d'un protocole de recherche répondant à une hypothèse, nous pensons que les pharmaciens doivent profiter du réseau EQUATOR $^{3}$. Le réseau EQUATOR (Enhancing the QUAlity and Transparency Of health Research) est une initiative internationale qui tient à jour une liste de toutes les lignes directrices suggérées ou exigées dans le cadre de la publication scientifique dans le domaine de la santé. Cette initiative a profité du financement d'organismes britanniques (National Institute for Health Research du National Health Service, Medical Research Council et Chief Scientist Office), américain (Pan American Health Organization) et canadien (Instituts de recherche en santé du Canada).

Le site Internet (www.equator-network.org/) est mis à jour périodiquement et comporte au moins 265 grilles, articles pivots et lignes directrices. Connaissez-vous les lignes directrices, telles que MOOSE, ${ }^{4} \mathrm{CONSORT}^{5}$ et STROBE ${ }^{6}$ ? Les listes de vérification sont classées selon le type d'étude, les tests statistiques réalisés, les domaines de recherche ou procédures réalisées, ainsi que selon les sections types utilisées dans les articles scientifiques (p. ex. figures, discussion et conflits d'intérêts).

À notre avis, la consultation de ces grilles au moment de l'élaboration d'un protocole de recherche en pratique pharmaceutique peut contribuer à sa rédaction conforme et permet de limiter les nombreux pièges méthodologiques. Alors que la recherche clinique repose le plus souvent sur les essais contrôlés à répartition aléatoire, la recherche évaluative menée sur les pratiques pharmaceutiques fait appel à de nombreux types d'études, telles que les études observationnelles incluant les sondages et audits, et les études économiques. Le tableau 1 présente une sélection limitée de listes de vérifications utiles à la recherche en pratique pharmaceutique. En choisissant une liste de vérification appropriée, le pharmacien peut identifier les sections clés de son protocole, les éléments de contenus sur lesquels il doit statuer et les bonnes pratiques attendues. La liste de vérification complétée pourrait être jointe à un protocole soumis à un comité scientifique afin de détailler la qualité de la démarche. Nous pensons qu'il y a trop peu de recherche en pratique pharmaceutique. L'utilisation du réseau EQUATOR peut contribuer à de meilleurs travaux de recherche, à vous de vérifier!

\section{Références}

1. Bussières JF, Tanguay C, Lebel D. 10 ans de recherche au sein de l'Unité de recherche en pratique pharmaceutique [affiche]. Présentée à la Journée annuelle de recherche du CHUQ, le 21 juin 2012. Publié au : http://urppchusj.wordpress.com/2012/06/21/urppchusj/. Consulté le 4 décembre 2012.

2. Charrois TL, Durec T, Tsuyuki RT. Systematic reviews of pharmacy practice research: methodologic issues in searching, evaluating, interpreting, and disseminating results. Ann Pharmacother. 2009;43(1):118-22.

3. EQUATOR network [site web]. Oxford (UK) : Equator Network; 2007 [mis à jour regulièrement]. Publié au : www.equator-network.org/. Consulté le 25 octobre 2012.

4. Stroup DF, Berlin JA, Morton SC, Olkin I, Williamson GD, Rennie D, et al.; Meta-analysis Of Observational Studies in Epidemiology (MOOSE) group. Meta-analysis of observational studies in epidemiology: a proposal for reporting. JAMA. 2000;283(15):2008-12.

5. Schulz KF, Altman DG, Moher D; CONSORT Group. CONSORT 2010 statement: updated guidelines for reporting parallel group randomised trials. BMJ. 2010:340:c332.

Tableau 1. Listes de vérifications utiles à la recherche en pratique pharmaceutique

\begin{tabular}{|c|c|c|}
\hline Type d'étude & Directives & Liste de vérification \\
\hline Méta-analyse & $\begin{array}{l}\text { MOOSE : Meta-analysis Of Observational Studies } \\
\text { in Epidemiology }{ }^{4}\end{array}$ & 35 items \\
\hline $\begin{array}{l}\text { Revue systématique, } \\
\text { méta-analyse }\end{array}$ & $\begin{array}{l}\text { PRISMA : Preferred Reporting Items for Systematic } \\
\text { Reviews and Meta-Analyses } \\
\text { (www.prisma-statement.org/) }\end{array}$ & 27 items \\
\hline Essai randomisé contrôlé & $\begin{array}{l}\text { CONSORT : CONsolidated Standards Of Reporting } \\
\text { Trials }{ }^{5} \text { (www.consort-statement.org/) }\end{array}$ & 37 items \\
\hline $\begin{array}{l}\text { Cohorte, cas-contrôle, } \\
\text { transversal }\end{array}$ & $\begin{array}{l}\text { STROBE : Strengthening the Reporting of Observational } \\
\text { Studies in Epidemiology }{ }^{6} \text { (www.strobe-statement.org/) }\end{array}$ & 22 items \\
\hline Série de cas & Kempen $^{8}$ & 7 items \\
\hline Cas & Sorinola et al. ${ }^{9}$ & 9 items \\
\hline Sondage & SURGE : SUrvey Reporting GuidelinE ${ }^{10}$ & 38 items \\
\hline Sondage en ligne & $\begin{array}{l}\text { CHERRIES : Checklist for Reporting Results from } \\
\text { Internet E-Surveys }{ }^{11}\end{array}$ & 29 items \\
\hline $\begin{array}{l}\text { Entrevue, groupe de } \\
\text { discussion }\end{array}$ & $\begin{array}{l}\text { COREQ : COnsolidated criteria for REporting Qualitative } \\
\text { research }^{12}\end{array}$ & 32 items \\
\hline Amélioration de la qualité & $\begin{array}{l}\text { SQUIRE : Standards for Quality Improvement Reporting } \\
\text { Excellence }^{13} \text { (http://squire-statement.org/) }\end{array}$ & 50 items \\
\hline Anecdotes & $\begin{array}{l}\text { PHARMA : Publishing Histories of Adverse Reactions to } \\
\text { Medicaments Anecdotally }{ }^{14}\end{array}$ & 40 items \\
\hline Réactions indésirables & Kelly et al. ${ }^{15}$ & 39 items \\
\hline Revue de littérature & STARLITE $^{16}$ & 8 items \\
\hline
\end{tabular}


6. von Elm E, Altman DG, Egger M, Pocock SJ, Gotzsche PC, Vandenbroucke JP. Strengthening the Reporting of Observational Studies in Epidemiology (STROBE) statement: guidelines for reporting observational studies. BMJ. 2007;335(7624):806-8.

7. Moher D, Liberati A, Tetzlaff J, Altman DG, The PRISMA Group. Preferred Reporting Items for Systematic Reviews and Meta-Analyses: the PRISMA statement. BMJ. 2009;339:b2535.

8. Kempen JH. Appropriate use and reporting of uncontrolled case series in the medical literature. Am J Ophthalmol. 2011;151(1):7-10.e1.

9. Sorinola O, Olufowobi O, Coomarasamy A, Khan KS. Instructions to authors for case reporting are limited: a review of a core journal list. BMCMed Educ. 2004;4:4.

10. Bennett C, Khangura S, Brehaut JC, Graham ID, Moher D, Potter BK, et al. Reporting guidelines for survey research: an analysis of published guidance and reporting practices. PLoS Med. 2010;8(8):e1001069.

11. Eysenbach G. Improving the quality of Web surveys: the Checklist for Reporting Results of Internet E-Surveys (CHERRIES). J Med Internet Res. 2004;6(3):e34.

12. Tong A, Sainsbury P, Craig J. Consolidated criteria for reporting qualitative research (COREQ): a 32-item checklist for interviews and focus groups. Int J Qual Health Care. 2007;19(6):349-57.

13. Davidoff F, Batalden P, Stevens D, Ogrinc G, Mooney S; SQUIRE Development Group. Publication guidelines for quality improvement in health care: evolution of the SQUIRE project. BMJ. 2009;338:a3152.

14. Aronson JK. Anecdotes as evidence. BMJ. 2003;326(7403):1346.

15. Kelly WN, Arellano FM, Barnes J, Bergman U, Edwards RI, Fernandez AM, et al.; International Society for Pharmacoepidemiology; International Society of Pharmacovigilance. Guidelines for submitting adverse event reports for publication. Drug Saf. 2007;30(5):367-73.

16. Booth A. "Brimful of STARLITE": toward standards for reporting literature searches. J Med Libr Assoc. 2006;94(4):421-9,e205.

Cynthia Tanguay, B. Sc., M. Sc.

Coordonnatrice

Denis Lebel, B. Pharm., M. Sc., FCSHP

Adjoint

Jean-François Bussières, B. Pharm., M.Sc., FCSHP

Chef

Unité de recherche en pratique pharmaceutique

Centre hospitalier universitaire Sainte-Justine

Montréal (Québec)

Denis Lebel est aussi adjoint aux soins pharmaceutiques, à I'enseignement et à la recherche, Département de pharmacie, Centre hospitalier universitaire Sainte-Justine, Montréal (Québec). Jean-François Bussières est aussi chef du Département de pharmacie au Centre hospitalier universitaire Sainte-Justine, et professeur titulaire de clinique, Faculté de pharmacie, Université de Montréal, Montréal (Québec).

Divulgation d'intérêts concurrents: Aucun déclaré.

\section{CJHP Subscriptions 20I3 / Abonnements au JCPH 2013}

CSHP has introduced both Print and Print + Online pricing models for CJHP subscriptions. Print + Online CJHP is included as a benefit of CSHP membership. All prices are in Canadian funds.

La SCPH a établi une grille tarifaire pour l'abonnement à la copie imprimée du JCPH seulement et pour l'abonnement à la fois aux copies imprimée et électronique du journal. L'abonnement combiné est inclus dans les droits d'adhésion à la SCPH. Tous les prix sont en dollars canadiens.

\begin{tabular}{|l|l|l|}
\hline $\begin{array}{l}\text { Subscriber group / Groupe } \\
\text { d'abonnés }\end{array}$ & $\begin{array}{l}\text { Print only / Texte imprimé } \\
\text { seulement }\end{array}$ & $\begin{array}{l}\text { Print + Online copy } \\
\text { Texte imprimé } \\
\text { et exemplaire électronique }\end{array}$ \\
\hline $\begin{array}{l}\text { Nonmembers within Canada / } \\
\text { Non-membres au Canada }\end{array}$ & $\begin{array}{l}\$ 120.00 \text { per year, plus GST or HST } \\
120,00 \$ \text { par an, plus TPS ou TVH }\end{array}$ & $\begin{array}{l}\$ 160.00 \text { per year, plus GST or HST } \\
160,00 \$ \text { par an, plus TPS ou TVH }\end{array}$ \\
\hline USA / É.-U. & $\begin{array}{l}\$ 150.00 \text { per year } \\
150,00 \$ \text { par an }\end{array}$ & $\begin{array}{l}\$ 190.00 \text { per year } \\
190,00 \$ \text { par an }\end{array}$ \\
\hline Foreign / Étranger & $\$ 190.00$ per year & $\$ 230.00$ per year \\
& $190,00 \$$ par an & $230,00 \$$ par an \\
\hline
\end{tabular}

More details can be found at www.cjhp-online.ca. If you would like to purchase a subscription, please fill out our CJHP 2013 Subscription Application Form, which can be found on the CJHP website.

Please direct any comments or questions to Colleen Drake, Publications Administrator, at cdrake@cshp.ca.

Des détails supplémentaires sont fournis à www.cihp-online.ca. Si vous désirez vous abonner, veuillez remplir le formulaire d'abonnement au JCPH 2013. Vous pouvez l'obtenir en visitant le site Web du $\mathrm{JCPH}$. Pour tout commentaire ou toute question, veuillez vous adresser à Colleen Drake, agente des publications, en écrivant à cdrake@cshp.ca. 